\title{
Study on the Mechanism of Liuwei Dihuang Pill s Formula in Treating Parkinson's Disease Based on Network Pharmacology
}

\section{Dongtao Lin}

Guangdong Pharmaceutical University

\section{Yudan Zeng}

Guangdong Pharmaceutical University

\section{Deyu Tang}

Guangdong Pharmaceutical University

Yongming Cai ( $\sim$ ymbruce@qq.com)

Guangdong Pharmaceutical University https://orcid.org/0000-0002-5507-0693

\section{Research}

Keywords: Bioinformatics, Network pharmacology, Parkinson's disease, Liuwei Dihuang Pills

Posted Date: April 8th, 2021

DOI: https://doi.org/10.21203/rs.3.rs-401619/v1

License: (c) (i) This work is licensed under a Creative Commons Attribution 4.0 International License. Read Full License 


\section{Abstract}

Background: Parkinson's Disease (PD) is a common neurodegenerative disease in middle-aged and elderly people. Liuwei Dihuang Pill (LWDH Pills) has good effect on Parkinson's disease but the mechanism of action is not clear. Network pharmacology is the result of integrating the basic theories and research methods of medicine, biology, computer science, bioinformatics and other disciplines, which can systematically and comprehensively reflect the mechanism of drug intervention in disease network.

Methods: Obtained the main components and targets of herbs in LWDH Pills through Traditional Chinese Medicine Systems Pharmacology Database and Analysis Platform (TCMSP) database, and screen the active components of traditional Chinese medicine according to ADME; The PD-related targets were obtained from Gencards, OMIM, TTD, DRUGBANK databases. Used Jvenn to take the intersection of targets of LWDH Pills and PD-related targets, use the String platform to analyze protein interactions, construct a PPI network and explore potential protein functional modules in the network. The Metascape platform was used to performe KEGG pathway and GO Function enrichment analysis. Finally, the Cytoscape software was used to construct the drug-components-target network.

Results: After screening and de-weighting, 210 effective active ingredients of LWDH Pills, 204 drug targets, 4333 diabetic nephropathy disease targets, and 162 drug-disease targets were obtained by the intersection of Jvenn. GO and KEGG enrichment analysis showed that these targets are involved in neuron death, G protein-coupled amine receptor activity, reactive oxygen species metabolic process, membrane raft, MAPK signaling pathway, cellular senescence and other biological processes. Drug-components-target shows that the hub components of Liuwei Dihuang Pills were quercetin, Stigmasterol, kaempferol, and beta-sitosterol

Conclusion: LWDH Pill has the characteristics of multi-component, multi-target and multi-pathway for the treatment of PD. The hub components may be quercetin, Stigmasterol, kaempferol, and beta-sitosterol, and may be through pairing hub targets such as AKT1, VEGFA, IL6, etc. to regulate Neuron death, G protein-coupled amine receptor activity, reactive oxygen species metabolic process, membrane raft, MAPK signaling pathway, cellular senescence to play a role in the treatment of PD

\section{Introduction}

Parkinson's disease (PD) is a common neurological disorder in the middle and old age, characterized by progressive degeneration of dopaminergic neurons in the substantia nigra and pathological changes in the formation of lewy bodies, biochemical changes in the decrease of dopamine (DA) transmitters in the striatum region, imbalance of dopamine and acetylcholine transmitters in the striatum region, motor symptoms such as tremor, myotonia, motor retardation, postural balance disorder and sleep disorders, olfactory disorders, autonomic nervous dysfunction, cognitive and mental disorders ${ }^{[1]}$. Epidemiological research shows that the prevalence of PD over 60 years old in Europe and the United States reaches $1 \%$, and over $4 \%$ are over 80 years old. The prevalence rate of people over 65 years old in China is $1.7 \%$, which is similar to European and American countries ${ }^{[2-3]}$. Drug therapy is the first choice for the treatment of PD. Levodopa is the standard treatment and the most effective symptomatic drug in the treatment of PD ${ }^{[4]}$. However, levodopa cannot completely cure Parkinson's disease, the long-term efficacy of the drug gradually worsens and brings a series of side effects ${ }^{[5]}$.

Traditional Chinese medicine (TCM) has more than 2,000 years of experience in treating Parkinson's disease. TCM believes that kidney yin deficiency is the key to the onset of Parkinson's disease. Liuwei Dihuang Pills (LWDH Pills) is a classic kidney-tonifying prescription created by Qian Yi, a famous doctor in the Northern Song Dynasty. Liuwei Dihuang Pills contains six herbal medicine: Rehmanniae Radix Praeparata (Shudihuang, SDH), Rhizoma Dioscoreae (Shanyao, 
SY), Cornus Officinalis Sieb (Shanzhuyu, SZY), Poria Cocos, (Fuling, FL), Cortex Moutan (Mudanpi, MDP), Alisma Orientale (Zexie, ZX). Clinical practice has proved that Liuwei Dihuang Pills was effective in treating PD, it can improve autonomic symptoms in patients with $\mathrm{PD}^{[6-7]}$. However, the six traditional Chinese medicines in Liuwei Dihuang Pills have complex chemical components, and their targets and mechanisms for the treatment of PD are not clear.

Network pharmacology (NP) was first proposed by British scholar Andrew L. Hopkins in 2007. NP is based on the rapid development of systems biology and multi-directional pharmacology. The new idea of drug design, which can expand the available drugs. Target space is one of the new strategies for new drug discovery ${ }^{[8]}$. It is based on the "disease-genetarget-drug" interaction network foundation, through the analysis of genes, proteins, diseases, drugs and other information in existing databases, combined with existing literature data, the use of professional network to build analysis software and structural biology Methods such as science and computational chemistry systematically and comprehensively reveal the intervention and influence of drugs on the disease network, thereby revealing the secret of the synergistic effect of drugs on the human body [9]. The holistic and systematic characteristics of its research strategy have the same goal as the theory of traditional Chinese medicine in diagnosing and treating diseases from a holistic perspective. It is very suitable for studying the relationship between various drug components and disease targets in traditional Chinese medicine. Thus, NP is widely used in the research of Chinese medicine. LI ${ }^{[10]}$ used HERB BIOMAP data collection, target map clustering, network target analysis and other methods to determine the anti-diabetic activity of the traditional Chinese medicine Gegen Qinlian Decoction. 4-Hydroxymethylphenytoin increased insulin secretion in RIN-5F cells and improved 3T3-L1 fat cells. Insulin resistance lays the foundation for the treatment of diabetes in the future. Zhang ${ }^{[11]}$ through molecular docking and network analysis, clarified the main active compounds, targets of action and various pharmacological mechanisms of Reduning injection in the treatment of upper respiratory tract infections, including inhibiting virus replication and directly acting on the key to regulating the life cycle of respiratory viruses Protein, indirectly regulates the host immune system, etc. ZENG Q et al. ${ }^{[12]}$ conducted network pharmacological analysis on Chaihu Shugan Powder and screened out 152 active ingredients, predicting that sapogenin F, sapogenin G, sapogenin C, leucoflorin, hesperidin, and hesperidin have good Through the enrichment analysis of GO and KEGG, it was found that it reduces Abeta-induced neuronal cell death and PC12 cell apoptosis through the PI3K-AKT signaling pathway, suggesting that it may have a therapeutic effect on Alzheimer's disease.

\section{Materials And Methods}

\section{Collection of Compounds and Target Prediction of LWDH Pills}

Traditional Chinese Medicine Systems Pharmacology Database and Analysis Platform (TCMSP, https://tcmspw.com/tcmsp.php) is an open source database that specializes in analyzing traditional Chinese herbal medicines, which integrates pharmacodynamics, pharmacokinetics, target prediction, and genomics ${ }^{[13]}$. We obtained compounds of each herbals in LWDH Pills from TCMSP platform with the search terms "Shudihuang", "Shanyao", "Shanzhuyu”, “Fuling”, “Mudanpi”, "Zexie”. Before targets prediction, used absorption, distribution, metabolism, and excretion (ADME) to select biologically active compounds that contribute to its therapeutic effect, while those with poor pharmacological properties and poor drug capabilities are removed ${ }^{[14]}$. We chose oral bioavailability $(\mathrm{OB}) \geq 30 \%$, and drug-likeness $(\mathrm{DL}) \geq 0.18$ as the ADME parameters. The target of LWDH Pill was also obtained through the TCMSP platform, The selected active compounds were collected in respective target databases to relate to their target.

\section{Predicting target of PD}

We obtained the PD-related target from four databases: (1) Durg Bank (https://go.drugbank.com/. Version 5.1.8) ${ }^{[15]}$, which is a comprehensive, free-to-access, online database containing information on drugs and drug targets. As both a bioinformatics and a cheminformatics resource. (2) Therapeutic Target Database (TTD, http://db.idrblab.net/ttd/, 
Updated June $1 \mathrm{st}, 2020)^{[16]}$, it is a database to provide information about the known and expected therapeutic protein and nucleic acid targets, the targeted disease, pathway information and the corresponding drugs directed at each of these targets. (3) Gene Cards (https://www.genecards.org/, Version 5.0) ${ }^{[17]}$, which is an online database that provides detailed information about all genes that have been annotated and predictable by humans, and automatically integrates gene-centric data from about 100 data sources. Including genome, transcriptome, proteome, genetics, clinical and functional information. (4) Online Mendelian Inheritance in Man (OMIM, https://omim.org/. Updated January 19, 2021) [18], it isis a comprehensive, authoritative compendium of human genes and genetic phenotypes that is freely available and updated daily. The full-text, referenced overviews in OMIM contain information on all known mendelian disorders and over 15,000 genes. We used "Parkinson's disease" as a keyword to screen disease targets in each database, and summarize the obtained targets and remove duplicate values. Finally, use the Uniprot database to standardize the obtained targets.

\section{Protein-Protein Interaction Network Construction and Module Screening}

In order to clarify the interaction between the related targets of Liuwei Dihuang Pills and the PD-related targets, Jvenn (http://jvenn.toulouse.inra.fr/app/index.html) was used to take the intersection of them and draw the Venn diagram to obtain drug-disease, which were the targets of Liuwei Dihuang Pill in the treatment of Parkinson's disease. STRING (https://string-db.org/, Version 11.0 ) is a database of known and predicted protein-protein interactions ${ }^{[19]}$. The interactions include direct (physical) and indirect (functional) associations; they stem from computational prediction, from knowledge transfer between organisms, and from interactions aggregated from other (primary) databases. We submitted the target in the intersection to the STRING to identify protein-protein interaction information, and use Cytoscape (Version 3.8.0) to visualize the network, which is an open source software platform for visualizing molecular interaction networks and biological pathways and integrating these networks with annotations, gene expression profiles and other state data ${ }^{[20]}$. Moreover, in order to more accurately analyze the action mechanism of LWDH Pills in treating dyslipidemia, it is necessary to further identify its important modules. The important modules and targets were screened from the PPI network with degree cutoff=2, max. Depth=100, k-core=2, and node score cutoff $=0.2$ by using the Molecular Complex Detection (MCODE) plug-in Cytoscape. P-value $\leq 0.05$ was considered as a significant difference..

\section{KEGG Pathway and GO Function Enrichment Analysis}

We applied Gene Ontology (GO) enrichment and Kyoto encyclopedia of genes and genomes (KEGG) pathway analysis to systematically analysed the biological functions of drug-disease targets ${ }^{[21-22]}$. Metascape (https://metascape.org/) ${ }^{[44]}$ can perform enrichment analysis on targets. which integrates multiple authoritative functional databases such as GO, KEGG, Uniprot, etc., and supports annotation, enrichment analysis. We submitted these targets to the Metascape to perform GO and KEGG Pathway enrichment analysis. P-value $\leq 0.01$ was considered as a significant difference.

\section{Construction and Analysis of drug-component-target Network}

Input the relationship between the active components of LWDH Pills and drug-disease targets into Cytoscape software to construct a drug-component-target network. Use the network analyzer analysis tool of Cytoscape software to analyze the network characteristic parameters, including Degree, Betweenness Centrality (BC) and ClosenessCentrality (CC), to screen the hub components and targets of LWDH Pill and the relationship between them.

\section{Results}

\section{Collection of Compounds and Target Prediction of LWDH Pills}


A total of 475 compounds of six herbs in LWDH Pills were obtained from TCMSP database. After the two key ADME parameters of $O B \geq 30 \%$ and $D L \geq 0.18$ are screened and duplicate values are removed, we got 210 active compounds, Including 19 compounds of FL, 32 compounds of MDP, 43 compounds of SY, 132 compounds of SZY, 2 compounds of $\mathrm{SDH}, 7$ compounds of ZX (figure 1). Finally, we obtained 204 targets of LWDH Pills through compiling the corresponding targets of active compounds of six herbs using TCMSP database.

\section{Predicting target of PD}

We searched the four databases of Genecards, OMIM, TTD, and DrugBank respectively, and the numbers of PD-related targets were $3827,526,89$, and 202 respectively. Summarize the obtained targets and remove duplicate values, and finally got 4333 PD-related targets.

\section{Protein-Protein Interaction Network Construction and Module Screening}

We submitted targets of LWDH Pills and PD-related targets to Jvenn to draw a Venn diagram (figure 2), and we obtained 162 drug-disease targets. Based on the analysis results of these drug-disease targets from STRING online database, we used Cytoscape software to constructed a PPI network with 160 nodes and 2800 edges (figure 3). We use the network analyzer analysis tool of Cytoscape software to analyze the network, and adjust the size of each target in the PPI network according to the degree value. The color of edges is based on the combined score between the targets, the larger the combined score, the darker the color. After obtaining the PPI network, we use the MCODE plug-in CytoScape software to analyze the interaction relationship through the molecular complex detection algorithm, and obtain the module (figure 3). According to $P$ value, the biological processes with the 3 best scores in the Module are retained respectively to describe their functions (table 1).

Table 1 LWDH Pills-PD targets PPI network function description

\begin{tabular}{|lll|}
\hline Module & Function description & $\log 10(P)$ \\
\hline Module1 & G protein-coupled amine receptor activity & -20.7 \\
\hline Module2 & MAPK signaling pathway & -13.88 \\
\hline Module3 & apoptotic signaling pathway & -10.02 \\
\hline
\end{tabular}

\section{KEGG Pathway and GO Function Enrichment Analysis}

We use Metascape database to perform enrichment analysis on the above drug-disease-related targets, including GO biological process (BP), GO cellular component (CC), GO molecular function (MF) and KEGG pathways. Then, we saved the top 20 results of each item, and made bubble charts for further analysis (figure 4). It can be seen that these targets enrich on many biological processes, and the LWDH Pills preparation can regulate various biological processes in the body to achieve the purpose of treating PD. Among them, the most closely related biological processes include neuron death, $\mathrm{G}$ protein-coupled amine receptor activity, reactive oxygen species metabolic process, membrane raft, MAPK signaling pathway, cellular senescence.

\section{Construction and Analysis of Drug-Component-Target Network}

According to the screened drug-disease targets and their pairing relationship with active ingredients, 23 active components of 210 active ingredients can be targeted to PD-related targets (table 2). Input the relationship between these active components and drug-disease target into Cytoscape software, we got drug-component-target network with 186 nodes and 379 edges (figure 5). We use the network analyzer analysis tool of Cytoscape software to analyze the 
network characteristic parameters to get Betweenness Centrality (BC), ClosenessCentrality(CC), Degree of each component. We predicted that quercetin $(B C=0.70528, C C=0.61056$, Degree $=117)$ is the main component of Liuwei Dihuang Pills in the treatment of Parkinson's disease, followed by Stigmasterol $(B C=0.08635, C C=0.38144$, Degree= 68), kaempferol $(B C=0.13884, C C=0.41761$, Degree $=45)$, and beta-sitosterol $(B C=0.10312, C C=0.39278$, Degree= 31).

Table 2 Characteristic parameters of network nodes for the main active ingredients of LWDH Pills 


\begin{tabular}{|c|c|c|c|c|c|}
\hline Mol ID & molecule name & $\begin{array}{l}\text { Attribution } \\
\text { Herbs }\end{array}$ & $\begin{array}{l}\text { Betweenness } \\
\text { Centrality }\end{array}$ & ClosenessCentrality & Degree \\
\hline MOL000098 & quercetin & MDP & 0.70528 & 0.61056 & 117 \\
\hline MOL000449 & Stigmasterol & SY,SZY,SDH & 0.08635 & 0.38144 & 68 \\
\hline MOL000422 & kaempferol & MDP & 0.13884 & 0.41761 & 45 \\
\hline MOL000358 & beta-sitosterol & SZY & 0.10312 & 0.39278 & 31 \\
\hline MOL000296 & hederagenin & $\mathrm{FL}$ & 0.03611 & 0.36926 & 16 \\
\hline MOL000322 & Kadsurenone & sY & 0.04245 & 0.36926 & 16 \\
\hline MOL000546 & diosgenin & SY & 0.05697 & 0.36634 & 14 \\
\hline MOL005430 & hancinone C & SY & 0.03033 & 0.36489 & 13 \\
\hline MOL001559 & piperlonguminine & SY & 0.01517 & 0.35783 & 8 \\
\hline MOL005465 & AIDS180907 & SY & 0.02240 & 0.35645 & 7 \\
\hline MOL005440 & Isofucosterol & SY & 0.02585 & 0.35509 & 6 \\
\hline MOL005530 & Hydroxygenkwanin & SZY & 0.00230 & 0.35509 & 6 \\
\hline MOL000492 & $(+)$-catechin & MDP & 0.00614 & 0.35373 & 5 \\
\hline MOL001736 & $(-)$-taxifolin & SY & 0.00070 & 0.35238 & 4 \\
\hline MOL002879 & Diop & SZY & 0.00091 & 0.35238 & 4 \\
\hline MOL007374 & $\begin{array}{l}\text { 5-[[5-(4-methoxyphenyl)-2- } \\
\text { furyl]methylene]barbituric acid }\end{array}$ & MDP & 0.01481 & 0.35238 & 4 \\
\hline MOL001494 & Mandenol & SZY & 0.00027 & 0.35104 & 3 \\
\hline MOL000273 & $\begin{array}{l}(2 \mathrm{R})-2- \\
{[(3 \mathrm{~S}, 5 \mathrm{R}, 10 \mathrm{~S}, 13 \mathrm{R}, 14 \mathrm{R}, 16 \mathrm{R}, 17 \mathrm{R})-3,16-} \\
\text { dihydroxy-4,4,10,13,14- } \\
\text { pentamethyl-2,3,5,6,12,15,16,17- } \\
\text { octahydro-1H- } \\
\text { cyclopenta[a]phenanthren-17-yl]-6- } \\
\text { methylhept-5-enoic acid }\end{array}$ & $\mathrm{FL}$ & 0.00273 & 0.34972 & 2 \\
\hline MOL001495 & Ethyl linolenate & SZY & 0.00013 & 0.34972 & 2 \\
\hline MOL002464 & 1-Monolinolein & $z x$ & 0.00013 & 0.34972 & 2 \\
\hline MOL003137 & Leucanthoside & SZY & 0.01081 & 0.34972 & 2 \\
\hline MOL005481 & $\begin{array}{l}\text { 2,6,10,14,18-pentamethylicosa- } \\
2,6,10,14,18 \text {-pentaene }\end{array}$ & SZY & 0.00004 & 0.34972 & 2 \\
\hline MOL005503 & Cornudentanone & SZY & 0.00004 & 0.34972 & 2 \\
\hline
\end{tabular}

\section{Discussion}

PD is a common neurodegenerative disease in middle-aged and elderly people. Because of its high prevalence, high disability rate, and chronic disease course, it has gradually become an important science and social issue in the field of population and health. DA receptor dysregulation is the pathophysiological basis of PD.However, there are still many confusions in its pathogenesis. It is currently believed that factors such as mitochondrial dysfunction caused by 
oxidative stress, abnormal protein folding caused by endoplasmic reticulum stress, neuroinflammation, microbiota-gutbrain axis and related gene changes are closely related to the occurrence and development of PD ${ }^{[23]}$.

In this study, we collected the targets of LWDH Pills and the targets of PD from multiple databases, combined them to obtain 162 targets of Liuwei Dihuang Pills for the treatment of PD, then we constructed PPI network and screening module. By observing the network, we can know that the important genes are AKT1, VEGFA, IL6. Basic research shows that AKT1 regulates pathological angiogenesis, vascular maturation and permeability in vivo ${ }^{[24]}$. VEGFA is a highly specific vascular endothelial cell growth-promoting factor, which can promote the increase of vascular permeability, the degeneration of extracellular matrix, the migration, proliferation and angiogenesis of vascular endothelial cells ${ }^{[25]}$. IL6 is a pleiotropic cytokine with a wide range of functions. IL- 6 can regulate the growth and differentiation of a variety of cells, has the function of regulating immune response, acute phase response and hematopoietic function, and plays an important role in the body's anti-infection immune response ${ }^{[26]}$. Furthermore, we screened the module in the PPI and described its function. The module is a potential subject of the PPI network. The density of subnet connections is high, and there are few connections in the area. Therefore, the module is considered to be a collection of biological significance. This collection has two meanings. One is a protein complex (protein complex). ), that is, multiple proteins work together to form a complex and then play a biological role; one is a functional module, such as proteins located in the same pathway, which interact more closely ${ }^{[27]}$. The results showed that the function of modules is related to $\mathrm{G}$ protein-coupled amine receptor activity, MAPK signaling pathway, and apoptosis signaling pathway. Previous studies have shown that DA receptors belong to the family of G protein-coupled receptors (GPCRs), DA receptors are regulated by $\mathrm{G}$ protein coupled receptor kinases (GRKs) and arrestins. Research on specific gene knockout mice suggests that GPCRs may be selectively phosphorylated by certain subtypes of GRKs and also selectively bind to certain arrestins subtypes $^{[28-29]}$. In the striatum, the D1 receptor mostly binds to arrestin3, while the D2 receptor mostly binds to $\operatorname{arrestin}^{[30-31]}$. Neuroinflammation is one of the important causes of DA neuron degeneration. Basic research showed that p38 mitogen-activated protein kinase (p38MAPK) signaling pathways are also involved in the immune inflammatory response of PD. And the apoptosis signaling pathway is one of the pathogenesis of PD that scholars currently consider.

Subsequently, we performed KEGG pathway and GO function enrichment analysis on these targets. According to q-value, the more important ones are neuron death, G protein-coupled amine receptor activity, reactive oxygen species metabolic process, membrane raft, MAPK signaling pathway, cellular senescence. Some of them are the same as the biological function of modules. Furthermore, neuron death is the pathological basis of PD. The reactive oxygen species metabolic process and membrane raft may be related to mitochondrial dysfunction and thus participate in the occurrence and development of PD. Astrocytes are the bridge connecting neurons and blood vessels, and are involved in activities such as underdevelopment, neurotransmitter transmission, brain metabolism, and blood flow regulation ${ }^{\text {[32-33] }}$. The maintenance of the mitochondrial respiratory chain function of astrocytes is very important for the energy balance of the brain and the production of antioxidants that protect neurons. HOEKSTRA et al. ${ }^{[34]}$ found that the expression of DRP1 in astrocytes in the brain of PD patients was reduced; knocking out DRP1 in astrocytes cultured in vitro significantly affected mitochondrial morphology and spatial positioning in astrocytes. It may interfere with the uptake of Ca2+coupled glutamate to produce hepatotoxicity and affect the survival of PD neurons. 1-methyl-4-phenyl-1,2,3,6tetrahydropyridine (MPTP) can be used in U373 MG human star glue Accumulate in the mitochondria of tumor cells, inhibit the synthesis of mitochondrial respiratory chain complex I, causing severe symptoms of PD ${ }^{[35]}$.

Finally, we constructed and analyzed the drug-component-target network to predict the hub component of LWDH Pills in the treatment of Parkinson's disease and their mechanisms. According to the degree, BC, and CC of the drug-componenttarget network, we predict that quercetin, Stigmasterol, kaempferol, and beta-sitosterol are the hub components of LWDH Pills. Quercetin has an antioxidant effect and can prevent cell damage induced by oxidative stress. Quercetin can effectively inhibit the transcription activity of cyclooxygenase 2 promoter, which can catalyze the production of 
arachidonic acid into prostaglandins (PGs) and other inflammatory substances, stimulate cell proliferation. Quercetin has a certain anti-inflammatory activity, and its anti-inflammatory effect is related to its strong oxidative effect [36]. Through metabolism in the body, the metabolites produced by quercetin have important anti-inflammatory effects ${ }^{\text {[37] }}$. Stigmasterol has anti-inflammatory and antioxidant effects, Panda et al. ${ }^{[38]}$ found that stigmasterol can reduce blood glucose concentration by reducing the release of thyroxine and increasing the concentration of insulin in the blood. At the same time, by reducing liver lipid peroxidation, increase the activity of catalase (CAT), Superoxide dismutase (SOD), glutathione (GSH) to exert its antioxidant activity. Pandith et al. ${ }^{[39]}$ found that stigmasterol has anti-inflammatory effects, which can significantly reduce the inflammatory factor cycloxygenase-2 (COX-2) and inducible nitric oxide synthesis stimulated by lipopolysaccharide (LPS). Enzyme (iNOS) mRNA expression, while exerting its anti-inflammatory effect by reducing the release of PGE2 and NO. Basic research shows that computers have anti-oxidant, anti-tumor, antiinfection and other biological activities. Moreover, Wu Can ${ }^{[40]}$ showed through animal experiments that campers will have a certain effect on the structure of mitochondria, and at the same time it will also have an inhibitory effect on the mitochondrial respiratory chain. Beta-sitosterol has anti-inflammatory, anti-oxidant and promotes the proliferation and

differentiation of embryonic neural stem cells. Yin et al. ${ }^{[41]}$ reacted Beta-sitosterol with organic acids to generate the derivative Beta-sitosterol-2-naphthoyl ester, and found that it can inhibit the expression of TLR4 and NF-KB to cause SOD in mice with acute liver injury, GSH level increases, MDA content decreases, and the same time enhances the expression of NRF-2 and HO-1 to inhibit oxidative stress. Liao et al. ${ }^{[42]}$ beta-sitosterol can inhibit the production of CAS1 and the activation of the MAPK signaling pathway by inhibiting the activation of the inflammasome NLRP3 in epidermal cells and macrophages, leading to TNF-a, IL-1beta, and MAPK signaling pathways in cells. The production of IL- 6 and IL8 is significantly reduced, thus playing an anti-inflammatory effect. Furthermore, beta-sitosterol in the diet can smoothly pass through the blood-brain barrier and be deposited on the brain cell membrane. Mahmoudi et al. ${ }^{[43]}$ found that after treating embryonic neural stem cells with beta-sitosterol-containing Alyssum hologram, the expression of NOTCH1, HES1, KI-67, and NICD proteins were significantly up-regulated, which promoted the proliferation and differentiation of embryonic neural stem cells.

\section{Conclusion}

In summary, based on network pharmacology technology and methods, this study explained the effective active ingredients of LWDH Pills and their related targets and pathways for the treatment of PD, and explained its multicomponent, multi-target, and multi-component treatment for PD. The hub components may be quercetin, Stigmasterol, kaempferol, and beta-sitosterol, and may be through pairing core targets such as AKT1, VEGFA, IL6, etc. to regulate Neuron death, G protein-coupled amine receptor activity, reactive oxygen species metabolic process, membrane raft, MAPK signaling pathway, cellular senescence to play a role in the treatment of PD. That provides a reference for in-depth exploration of the pharmacological effects of LWDH Pills. Because some bioinformatics data inventory is limited, it cannot contain all the active ingredients and targets in LWDH Pills, so this article still has certain limitations. The next step is to use clinical or animal experiments for deeper exploration.

\section{Declarations}

\section{Ethics approval and consent to participate}

Not applicable.

\section{Consent for publication}

Not applicable. 
Availability of data and materials

The datasets obtained from TCMSP, Durg Bank, Gene Cards, TTD, OMIM.

\section{Competing interests}

The authors declare that there is no potential conflicts of interest.

\section{Funding}

The Project-sponsored by SRF for ROCS, SEM and supported by the Project of Chinese Ministry of Education (N0.2017A11001),Research on Prediction Trend of Population Infected with COVID-19 Based on Big Data (2020KZDZX1126)

\section{Authors' contributions}

All authors contributed to study conception and design, Lin Dongtao is responsible for data collection. Analysis of data and visualization of results were conducted by writing Dongtao Lin and Yudan Zeng. The manuscript Was written by Dongtao Lin. Deyu Tang, Yongming Cai were responsible for revising the manuscript. All authors read and approved the final manuscript.

\section{Acknowledgements}

The results of this study are based on the data from TCMSP,Durg Bank, Gene Cards, TTD, OMIM. We thank the authors who provided the data for this study.

\section{References}

1. Kalia L V , DAE Lang. Parkinson's disease[J]. Lancet, 2015, 386(9996):896-912.

2. Lo R Y . Parkinson's Disease; Epidemiology[J]. Encyclopedia of the Neurological Sciences, 2014:833-839.

3. Parkinson's Disease and Movement Disorders Group of Neurology Branch of Chinese Medical Association. Guidelines for the Treatment of Parkinson's Disease in China (Fourth Edition) [J]. Chinese Journal of Neurology, 2020, 53(12): 973-986.

4. Connolly B S , Lang A E . Pharmacological Treatment of Parkinson Disease: A Review[J]. Jama, 2016, 311(16):16701683.

5. Schapira A, Emre M , Jenner $P$, et al. Levodopa in the treatment of Parkinson's disease[J]. European Journal of Neurology, 2010, 16(9).

6. Fang Ling, Fan Yongping, Wang Lei. Study on the clinical application of Liuwei Dihuang Pills (decoction)[J]. Chinese Journal of Traditional Chinese Medicine, 2013, 28(07): 2181-2185.

7. Wang Di, Shang Xiaoling. Liuwei Dihuang Pills to prevent and treat senile diseases[J]. Chinese Journal of Gerontology, 2011, 31(03): 524-527.

8. Hopkins A L. Network pharmacology[J].Nat Biotechnol,2007,25(10):1110

9. Jiang W , Y Bao, Guangli D U . Research Progress of Network Chinese Pharmacology. Information on Traditional Chinese Medicine, 2018.

10. Li H, Zhao L , Bo Z , et al. A Network Pharmacology Approach to Determine Active Compounds and Action Mechanisms of Ge-Gen-Qin-Lian Decoction for Treatment of Type 2 Diabetes[J]. Evidence-Based Complementray and Alternative Medicine,2014,(2014-1-16), 2014, 2014:495840. 
11. Zhang X, Gu J, Cao L, et al. Network pharmacology study on the mechanism of traditional Chinese medicine for upper respiratory tract infection [J]. Mol Biosyst,2014, 10(10): 2517-2525.

12. Zeng Q , Li L , Siu W, et al. A combined molecular biology and network pharmacology approach to investigate the multi-target mechanisms of Chaihu Shugan San on Alzheimer's disease[J]. Biomedicine \& Pharmacotherapy, 2019, 120:109370-.

13. Ru J , Li P, Wang J , et al. TCMSP: a database of systems pharmacology for drug discovery from herbal medicines[J]. J Cheminform, 2014, 6(1):13.

14. Hou T , Xu X . ADME evaluation in drug discovery[J]. Molecular Modeling Annual, 2002, 8(12):337-349.

15. X. Wang, S. Zhang, F. C. Li, Y. Zhou, Y. Zhang, R. Y. Zhang, J. Zhu, Y. X. Ren, Y. Tan, C. Qin, Y. H. Li, X. X. Li, Y. Z. Chen* and F. Zhu*. Therapeutic Target Database 2020: enriched resource for facilitating research and early development of targeted therapeutics. Nucleic Acids Research. 48(D1): D1031-D1041 (2020).

16. X. Wang, S. Zhang, F. C. Li, Y. Zhou, Y. Zhang, R. Y. Zhang, J. Zhu, Y. X. Ren, Y. Tan, C. Qin, Y. H. Li, X. X. Li, Y. Z. Chen* and F. Zhu*. Therapeutic Target Database 2020: enriched resource for facilitating research and early development of targeted therapeutics. Nucleic Acids Research. 48(D1): D1031-D1041 (2020).

17. Stelzer G , Rosen N , Plaschkes I, et al. The GeneCards Suite: From Gene Data Mining to Disease Genome Sequence Analyses[J]. Current Protocols in Bioinformatics, 2016, 54.

18. Amberger J S , Bocchini C A, Schiettecatte F , et al. OMIM.org: Online Mendelian Inheritance in Man (OMIM), an Online catalog of human genes and genetic disorders[J]. Nucleic Acids Research, 2014, 43(D1).

19. Damian S , Morris J H, Helen C , et al. The STRING database in 2017: quality-controlled protein-protein association networks, made broadly accessible[J]. Nucleic Acids Research, 2017:D362-D368.

20. Shannon, P. Cytoscape: A Software Environment for Integrated Models of Biomolecular Interaction Networks[J]. Genome Research, 2003, 13(11):2498-2504.

21. Blake J A , Chan J , Kishore R, et al. Gene Ontology Consortium: going forward[J]. Nucleic Acids Research, 2015, 43(Database issue):1049-56.

22. Gerlich M , Neumann S. KEGG: kyoto encyclopedia of genes and genomes.[J]. Nucleic Acids Research, 2000, 28(1):27-30.

23. Sumit S, James R, Syed I . Neuroprotective and Therapeutic Strategies against Parkinson's Disease: Recent Perspectives[J]. International Journal of Molecular Sciences, 2016, 17(6):904

24. Akt1 regulates pathological angiogenesis, vascular maturation and permeability in vivo.

25. Tammela T, Enholm B , Alitalo K, et al. The biology of vascular endothelial growth factors[J]. Cardiovascular Research, 2005, 65(3):550.

26. Xing Z . IL-6 is an antiinflammatory cytokine required for controlling local or systemic acute inflammatory responses.[J]. Journal of Clinical Investigation, 1998, 101(2):311-320.

27. Vella D , Marini S, Vitali F, et al. MTGO: PPI Network Analysis Via Topological and Functional Module Identification[J]. Scientific Reports, 2018, 8(1):5499.

28. Kohout, Trudy, A, et al. Beta-Arrestin 1 and 2 differentially regulate heptahelical receptor signaling and trafficking.[J]. Proceedings of the National Academy of Sciences of the United States of America, 2001, 98(4):1601-1601.

29. Oakley R H . Differential Affinities of Visual Arrestin, beta Arrestin1, and beta Arrestin2 for G Protein-coupled Receptors Delineate Two Major Classes of Receptors[J]. Journal of Biological Chemistry, 2000.

30. Macey T A , Liu Y , Gurevich V V , et al. Dopamine D1 receptor interaction with arrestin3 in neostriatal neurons[J]. Journal of Neurochemistry, 2010, 93(1):128-134.

Page $11 / 16$ 
31. Macey, T. A . Preferential Interaction between the Dopamine D2 Receptor and Arrestin2 in Neostriatal Neurons[J]. Molecular Pharmacology, 2004, 66(6):1635-1642.

32. Khakh B S , Sofroniew M V . Diversity of astrocyte functions and phenotypes in neural circuits[J]. Nature Neuroscience.

33. Verkhratsky A, Zorec R , Parpura V . Stratification of astrocytes in healthy and diseased brain[J]. Brain Pathology, 2017, 27(5):629-644.

34. Jake, G, Hoekstra, et al. Astrocytic Dynamin-Like Protein 1 Regulates Neuronal Protection against Excitotoxicity in Parkinson Disease[J]. American Journal of Pathology, 2015.

35. Huenchuguala Sandro and Muñoz Patricia and Segura-Aguilar Juan. The Importance of Mitophagy in Maintaining Mitochondrial Function in U373MG Cells. Bafilomycin A1 Restores Aminochrome-Induced Mitochondrial Damage. [J]. ACS chemical neuroscience, 2017, 8(10) : 2247-2253.

36. Sattu Kamaraj et al. The Effects of Quercetin on Antioxidant Status and Tumor Markers in the Lung and Serum of Mice Treated with Benzo(a)pyrene[J]. Biological and Pharmaceutical Bulletin, 2007, 30(12) : 2268-2273.

37. Loke Wai Mun et al. Metabolic transformation has a profound effect on anti-inflammatory activity of flavonoids such as quercetin: lack of association between antioxidant and lipoxygenase inhibitory activity.[J]. Biochemical pharmacology, 2008, 75(5) : 1045-53.

38. Panda, S, Jafri, et al. Thyroid inhibitory, antiperoxidative and hypoglycemic effects of stigmasterol isolated from Butea monosperma[J]. FITOTERAPIA -MILANO-, 2009, 80(2):123-126.

39. Pandith H, Zhang X, Thongpraditchote S, et al. Effect of Siam weed extract and its bioactive component scutellarein tetramethyl ether on anti-inflammatory activity through NF-kB pathway[J]. Journal of Ethnopharmacology, 2013, 147(2):434-441.

40. Wu Can. The effects of Morin, Paclitaxel and Kaempferol on isolated mitochondria[D]. Guangxi Normal University, 2017.

41. Yin Y , Liu X , Liu J , et al. Beta-sitosterol and its derivatives repress lipopolysaccharide/ d -galactosamine-induced acute hepatic injury by inhibiting the oxidation and inflammation in mice[J]. Bioorganic \& Medicinal Chemistry Letters, 2018:S0960894X18302786.

42. Pei-Chun, Liao, Ming-Hoang, et al. Identification of beta-Sitosterol as in Vitro Anti-Inflammatory Constituent in Moringa oleifera.[J]. Journal of Agricultural \& Food Chemistry, 2018.

43. Mahmoudi R, Ghareghani M , Zibara K, et al. Alyssum homolocarpum seed oil (AHSO), containing natural alpha linolenic acid, stearic acid, myristic acid and $\beta$-sitosterol, increases proliferation and differentiation of neural stem cells in vitro[J]. BMC Complementary and Alternative Medicine, 2019, 19.

44. Zhou Y , Zhou B , Pache L , et al. Metascape provides a biologist-oriented resource for the analysis of systems-level datasets[J]. Nature Communications, 2019, 10(1).

\section{Figures}



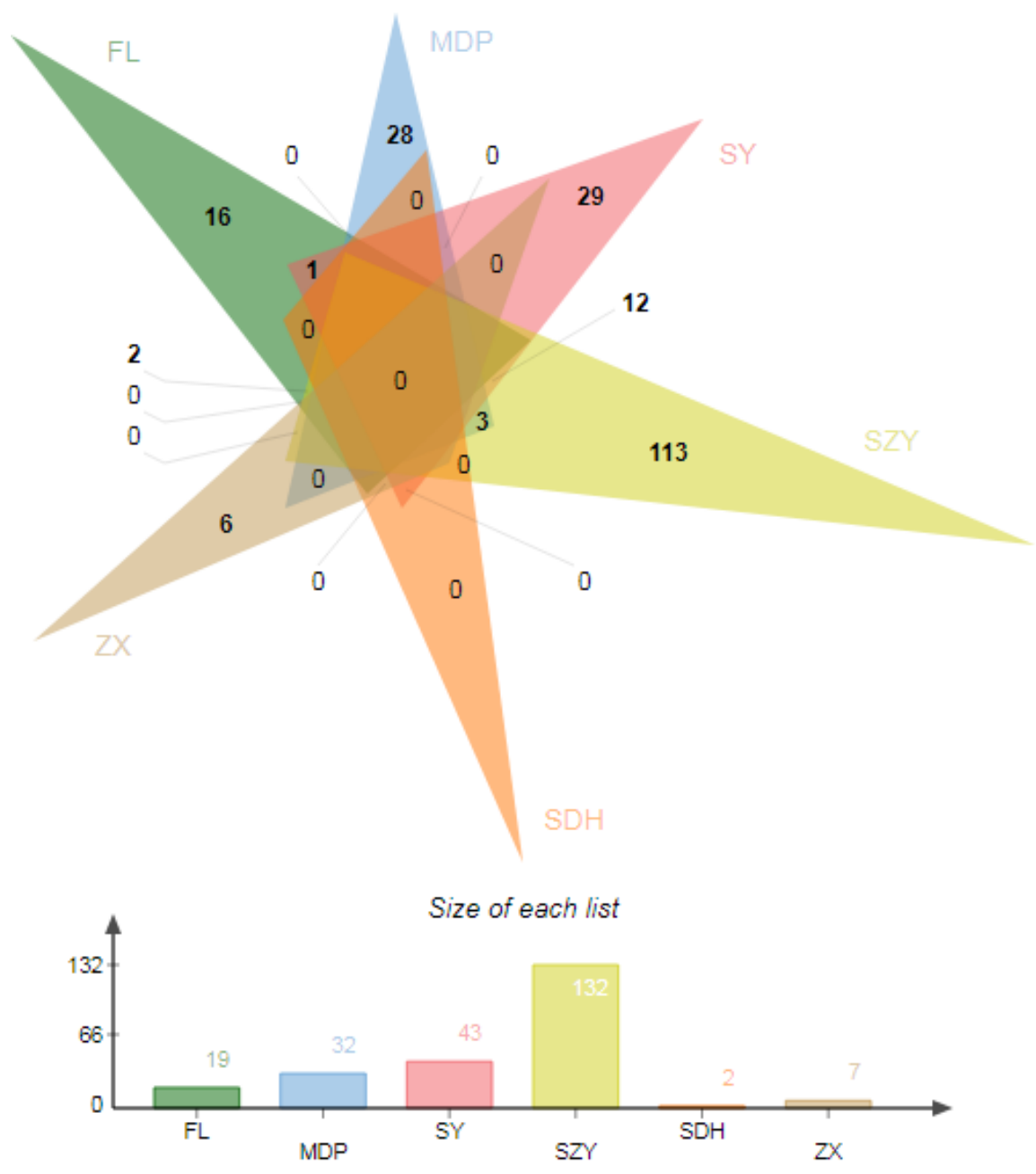

Number of elements: specific (1) or shared by $2,3, \ldots$ lists

\begin{tabular}{c||c|}
\hline 18 & 192 \\
\hline $4(12$ & 1 \\
$3(1)$ & 1
\end{tabular}

Figure 1

active compounds of six herbs in LWDH Pill 

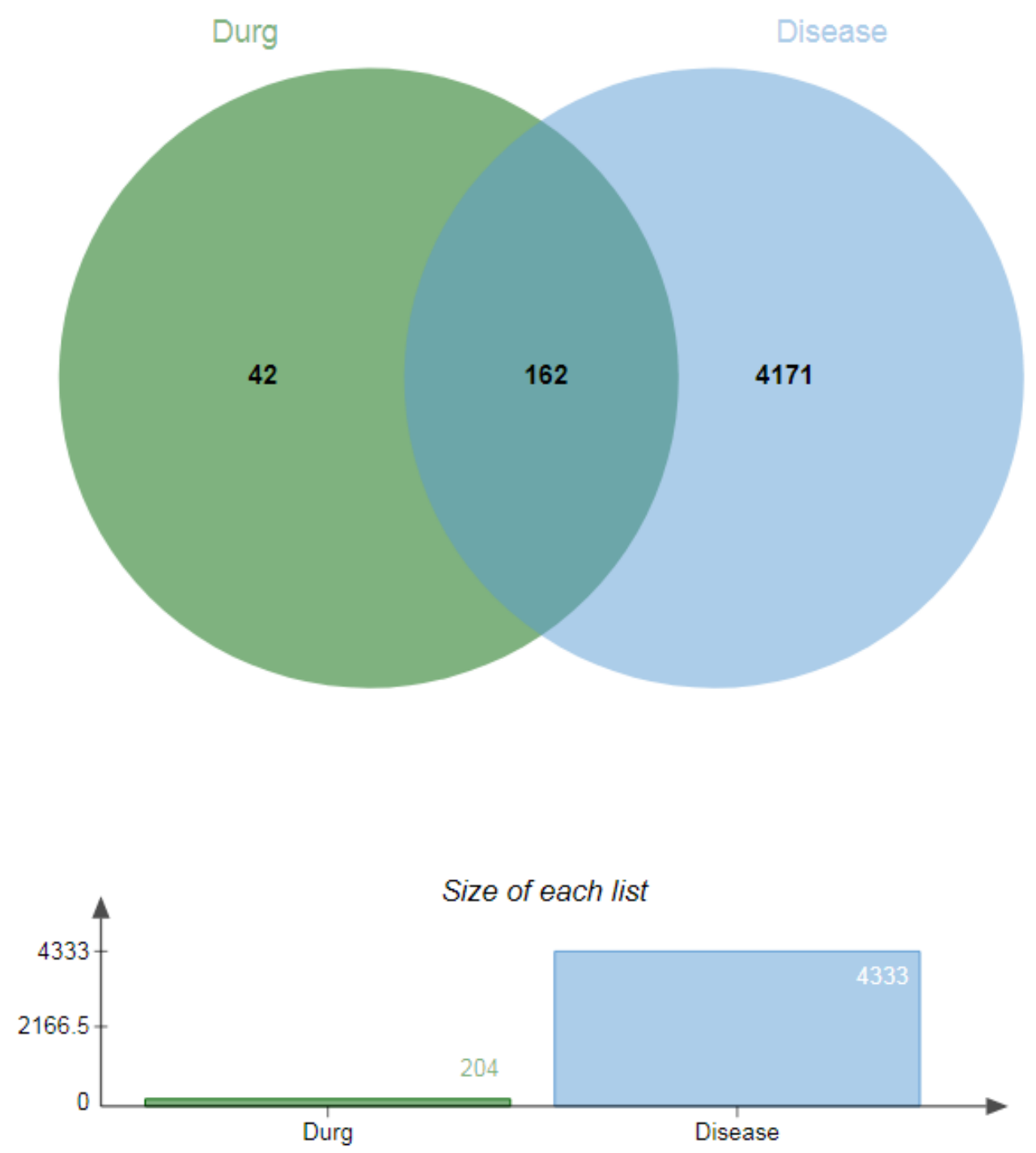

Number of elements: specific (1) or shared by 2, 3, .. lists

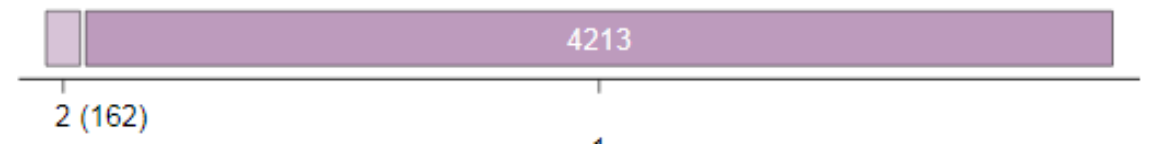

Figure 2

Venn diagram of PD-related targets and targets of LWDH Pills 


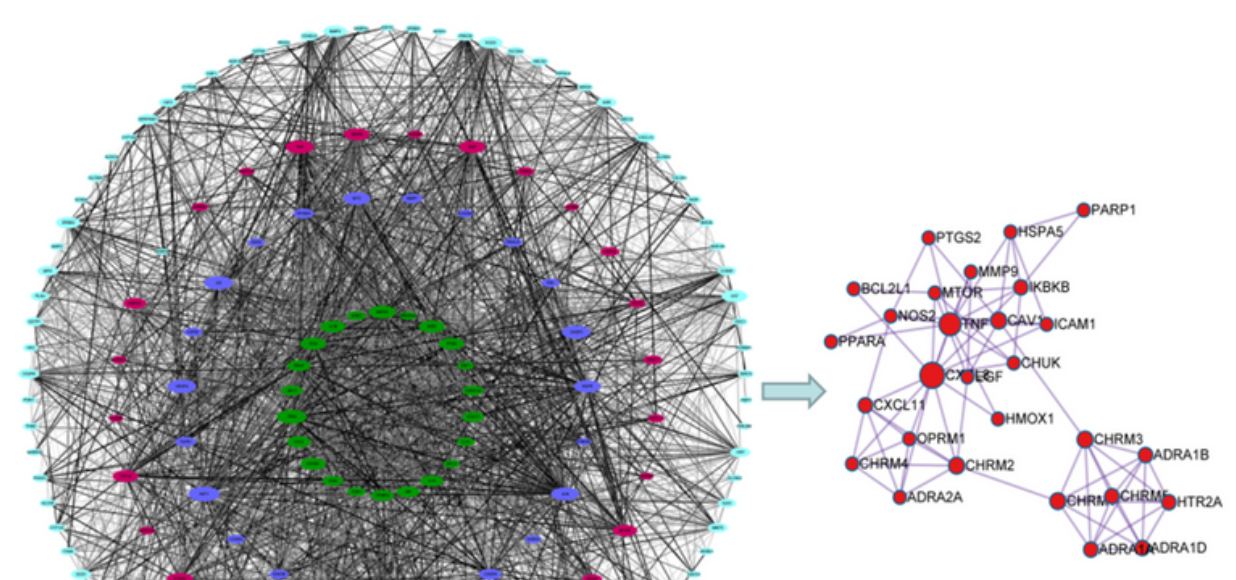

Module1

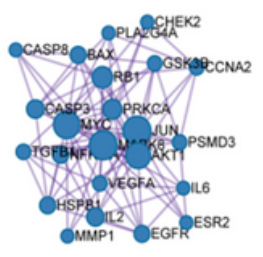

Module2

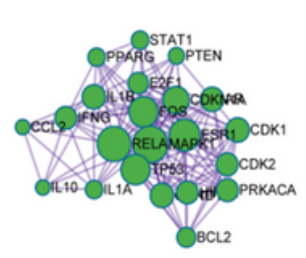

Module3

Figure 3

LWDH Pills-PD target PPI network and module. The nodes represents the target, the edges represents the matching short relation, and the size of nodes represents the degree, color of edges represents combined score.
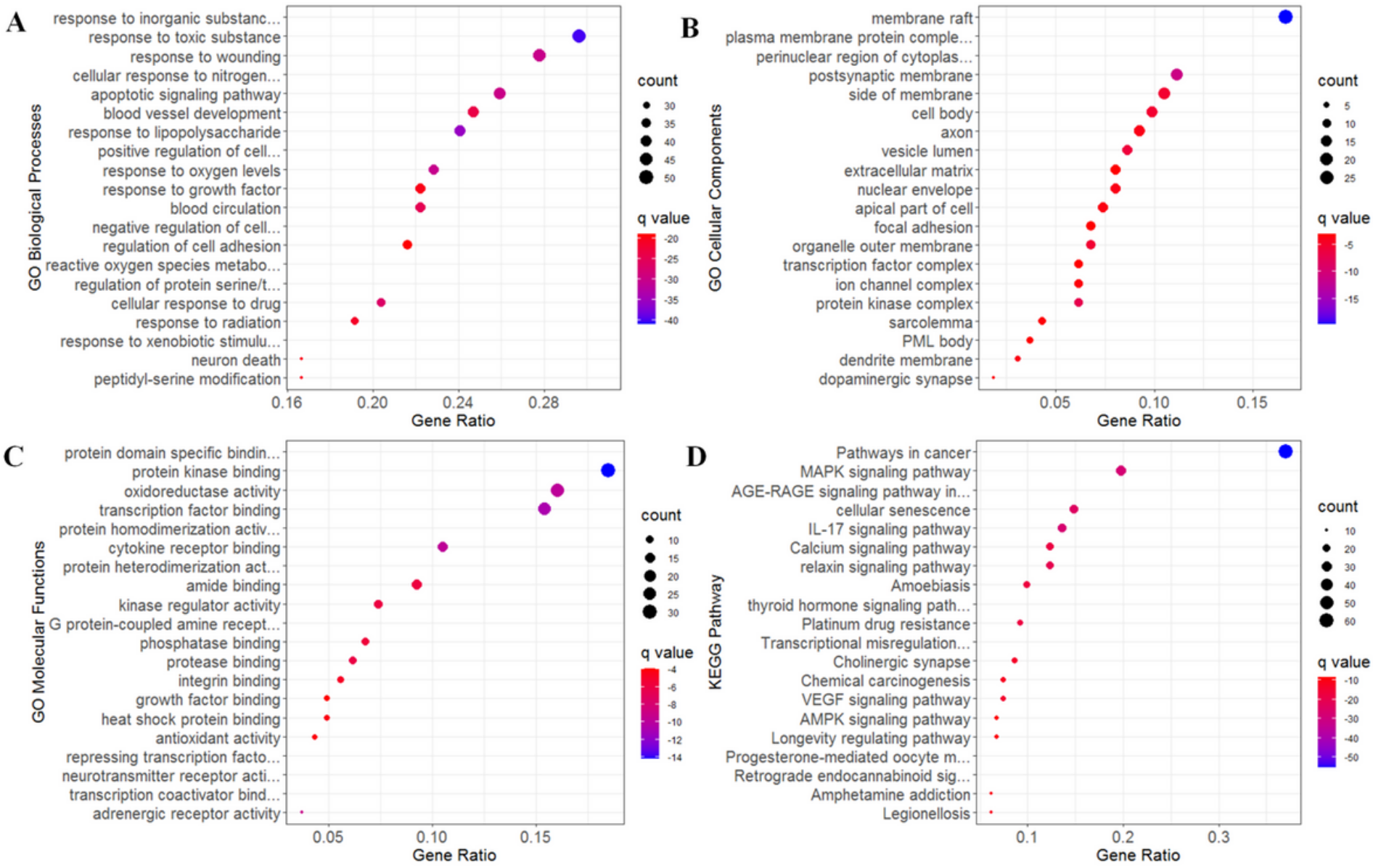

Figure 4

Results of enrichment analysis 


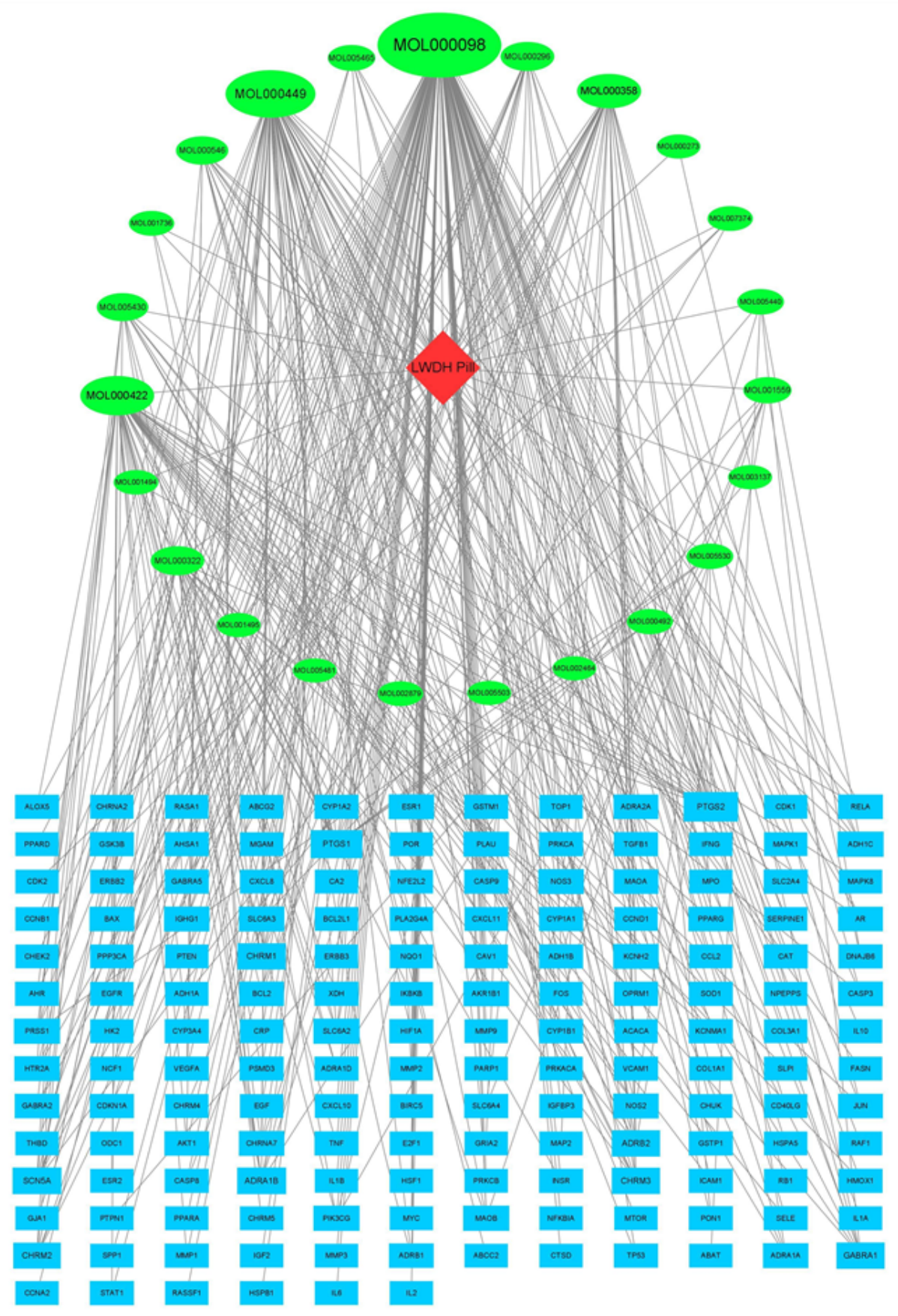

\section{Figure 5}

Drug-Components-Targest Network. The circular nodes represent the components, the rectangular nodesthe represent the drug-disease targets, the diamond node represents LWDH Pills, The edges represents the matching short relation, and the size of nodes represents the degree. 